\title{
Risk of Fall among Chronic Type 2 Diabetes Mellitus Patients in Lahore: A Case Series
}

\section{Zeeshan Saeed ${ }^{1}$, Shahzad Ahmed ${ }^{2}$, Samreen Sadiq ${ }^{3}$, Hafiz Muhammad Asim ${ }^{4}$}

${ }^{1}$ Senior Lecturer, Lahore College of Physical therapy, Lahore Medical \&Dental College

${ }^{2-3}$ Assistant Professor, Lahore College of Physical therapy, Lahore Medical \& Dental College

${ }^{4}$ Professor, Lahore College of Physical therapy, Lahore Medical \&Dental College.

Author's Contribution

1-2Conception and design, Collection and assembly of data, ${ }^{2-3}$ Analysis and interpretation of the data, ${ }^{3-4}$ Critical revision of the article for important intellectual content, Statistical expertise 1-4Final approval and guarantor of the article.

Article Info.

Received: Dec 2, 2019

Acceptance: Oct 13, 2021

Conflict of Interest: None

Funding Sources: None

Address of Correspondence

Dr Zeeshan Saeed

Email Id: Zeeshan.saeed@Imdc.edu.pk ORIC ID: 0000-0001-5556-2199

Cite this article as: Saeed Z, Ahmed S, Sadiq S, Asim HM. Risk of Fall among Chronic Type 2 Diabetes Mellitus Patients in Lahore: A Case Series. JRCRS. 2021; 9(2):87-91. DOI: 10.53389/JRCRS.2021090211

\section{A B S T R A C T}

Background: Individuals with diabetes have an incremental risk of developing a number of complications and among them, the most prevalent complication is, diabetic neuropathy causing sensory and motor deficits resulting in balance impairments and increases the risk of fall.

Objective: To determine the risk of fall in chronic type 2 diabetes mellitus.

Methodology: A descriptive case series was conducted at Diabetic Out-Patient Unit of Ghurki Trust Teaching Hospital, Lahore. 59 diagnosed cases of chronic type 2 diabetes mellitus were recruited based on inclusion criteria. Each participant was questioned before conducting an assessment. The inclusion criteria were established neuropathy and patients having diabetes mellitus for at least ten years. Exclusion criteria were patients with recent onset of diabetes, rheumatic and musculoskeletal diseases which interfere with daily activities, neurological conditions which affects balance (Parkinson's and Stroke) and severe pain. Data were recorded through interviews and balance test. Berg Balance Scale was used to assess the static and dynamic balance of the patients.

Results: Mean age of the patients was $57.43 \pm 7.28$ years. $19(32.2 \%)$ of the patients were male and $40(67.8 \%)$ patients were female. In a sample size of $59,17(28.8 \%)$ patients were at high risk of falling, whereas $33(55.9 \%)$ patients were at medium and $9(15.3 \%)$ patients were at low risk of fall respectively.

Conclusions: Greater numbers of patients with diabetic neuropathy have moderate risk of fall.

Keywords: Balance impairments, Berg balance scale, Diabetes Mellitus type 2, Diabetic Neuropathy, Fall Risk.

\section{Introduction}

Diabetes is a complex disease which progressively affects multiple systems of the body. Sensory and motor deficits resulting from diabetes leads to balance impairments and other

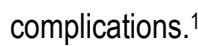

Lately, incidence of diabetes has witnessed an incremental trend. The International Diabetes Federation in 2019 estimated that approximately $9.3 \%$ of the world population had diabetes, with type II making up $90 \%$ of the cases. ${ }^{2}$ The prevalence of diabetes is high in Pakistan, approximately $14.62 \%$ individuals in $2019 .^{3}$
People with diabetes have an incremental risk of developing a number of complications and among them, the most prevalent complication is, diabetic neuropathy approximately $20-30 \%$ and up to $50 \%$ patients will eventually develop neuropathy along the course of their disease. ${ }^{4}$ Diabetic neuropathy is the debilitating complication which is consorting with considerable morbidity, mortality and reduced quality of life. ${ }^{5}$ The most common clinical manifestation includes pain and sensory loss with typical glove and stocking pattern, which is followed by consequential motor deficits as the disease progress. 
The loss of sensory perception secondary to diabetic neuropathy has a marked detrimental effect on balance; both static and dynamic. ${ }^{6}$ Balance is the skill which refers to center of gravity within the base of support. ${ }^{7}$ Balance control is a complex mechanism which involves the integration of somatosensory, visual and vestibular systems along with the structural and biomechanical components. ${ }^{8}$

During an unexpected perturbation, somatosensory input response provides the early stabilization control, whereas the visual and vestibular system contributes to later components of the postural control response. ${ }^{9}$ Impairments in somatosensory system secondary to neuropathy results in substantial deficiencies in balance control. ${ }^{10}$

Deterioration of proprioception and kinesthetic sensation secondary to diabetic neuropathy has marked detrimental effects on perception and stability, leading to impairments in locomotion. ${ }^{11}$ Deterioration of proprioceptive mechanisms resulting from diabetic neuropathy can escalate into faulty postural control mechanics including ankle, hip and stepping strategies and loss of coordination, which leads to impaired balance and increases the risk of falling. ${ }^{12}$

Decrement in balance and altered walking pattern secondary to sensory and motor deficits, is the major cause of falls in older adults with the diabetic neuropathy. Fall is considered as the major health issue among older adults with type 2 diabetes mellitus. The devastating consequences of falling include declines in mobility, activity constraints and reduced quality of life. Fall associated injuries are the leading cause of death and hospitalization in adults with diabetes ${ }^{(13)}$.Neuropathic changes alters the gait parameters and postural strategies ${ }^{(14)}$. Healthy adults rely on ankle strategy for maintenance of balance as compared to neuropathic individuals, who rely on hip strategy because of reduced proprioception around ankle joint to maintain the upright balance. When such patients are exposed to challenging environments the compensation strategies fail, and predisposes them to fall. 15

The Purpose of the study is to describe the impact of chronic type 2 diabetes mellitus on balance which will help provide a better quality of life to patient's through comprehensive treatment and preventive measures.

Methodology

The descriptive case series was conducted to evaluate the balance impairments in chronic type 2 diabetes mellitus patients. The study was carried out in the Diabetic OutPatient Unit of Ghurki Trust Teaching Hospital, Lahore, from March - August 2017. Diagnosed cases of chronic type 2 diabetes mellitus were recruited. Each participant was questioned regarding the history of onset and progression of symptoms before conducting an assessment, for inclusion and exclusion criteria. The inclusion criteria were age between 35 and 65 years, with established neuropathy and patients having diabetes mellitus for at least ten years. An exclusion criterion was patients with recent onset of diabetes, rheumatic and musculoskeletal diseases which interfere with daily activities, neurological conditions which affects balance (Parkinson's and Stroke) and severe pain. 59 patients with diabetes who met the inclusion criteria were recruited using non-probability convenience sampling. Data were recorded through semistructured interview which included open -ended questions such as history of diabetes, episodes of loss of balance, and falls. Formal assessment was done by balance test (BBS).

Berg Balance Scale was used to assess the static and dynamic balance of the patients, which has a high reliability $0.98^{(16)}$. There were no ethical issues in the study. An informed consent was obtained from each patient prior to test and study had been approved by the ethical committee of the Lahore college of Physical Therapy (LCPT) under reference number ERC-486-2017. Participants preformed each test of the Berg Balance Scale and the result was recorded as the score of the test. The balance assessment consists of 14 tasks performed in a standard order. Each task is scored on a 5-point scale 0-4, (0 - inability to complete the task, 4 - independent task fulfillment) according to the quality of the performance and the time taken to complete the task. The maximum score for this assessment is 56 . A score below 45 indicates impairment, with an increased risk for falls

\section{Results}

$19(32.2 \%)$ of the patients were male and $40(67.8 \%)$ patients were female. The mean age of patients was $53.47 \pm 7.28$ years. Out of 59 patients $46(78.0 \%)$ patients were suffering from the diabetes from the past 10-20 years and 13 $(22.0 \%)$ were those patients who had diabetes for 21-30 years. (Table I)

\begin{tabular}{lc}
\hline \multicolumn{2}{l}{ Table I: Demographic data of Diabetic Patients } \\
\hline $\begin{array}{l}\text { Demographics } \\
\text { characteristics }\end{array}$ & Frequency, Mean \pm S.D \\
\hline Age & $53.47(\mathbf{\pm 7 . 2 8 )}$. \\
\hline Gender & Male (19), Female (40). \\
\hline Duration of diabetes & $17.10(\mathbf{\pm 4 . 4 5 ) .}$ \\
\hline
\end{tabular}

Mean duration of diabetes was $17.10 \pm 4.45$ years. On the basis of berg balance scale result showed that $17(28.8 \%)$ patients were at high risk of falling, whereas 33 (55.9\%) patients were at moderate risk and $9(15.3 \%)$ patients were at 
low risk of fall respectively. The majority of the participants were at medium risk of fall who had diabetes for 10-20 years.

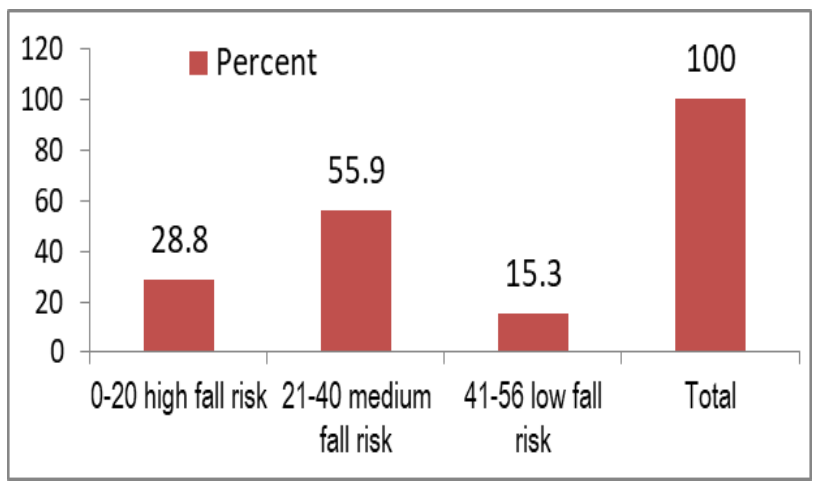

Figure 1. Balance impairments and associated fall risk.

$$
\text { Discussion }
$$

In the present study, balance was evaluated among participants with type 2 diabetes by the utilization of a validated tool for balance assessment known as berg balance scale. The results of the current study showed a poor functional balance in quarter of diabetic population considering the BBS overall score.

Diabetic neuropathy and associated loss of proprioception with other complications is the major entity for balance impairments. Thus a strong association can be identified between diabetic neuropathy and impairment in balance which consequently results in fall among such population. Another important aspect can be highlighted that there is also a direct proportionate relation between severity of neuropathy and balance impairment. ${ }^{17}$ The development of diabetic neuropathy is not only linked with poor glycemic control but also correlated with numerous other factors like the demographic characteristics of patients and associated comorbidities. However previous literature has emphasized the fact that severity of diabetic neuropathy is a strong predictor of balance impairments among diabetic cases. ${ }^{18}$

Loss of proprioceptive feedback from the lower extremity in diabetic neuropathy results in postural instability when the body is exposed to unexpected perturbations. Most of the previous studies assessed postural control during quite standing and their findings revealed significant changes in postural control parameters. ${ }^{19}$ Among BBS tests, single leg stance seems to be the most difficult task for DPN patients( ${ }^{(20)}$. Reduced ankle strategy in diabetic neuropathic patients result in postural instability and make this task difficult to perform. BBS tasks such as tandem standing and standing unsupported with feet together in which the base of support is reduced are more challenging for DPN participants. ${ }^{21}$ DPN participants showed worse performance in dynamic task in which COG moves while BOS is fixed such as reaching forward in the standing position, sitting to stand and standing to sit and conditions in which both $\mathrm{OOG}$ and BOS are changing transfers and placing the alternative foot on step or stool while standing unsupported tests.

The mean age of participants in our study was 53.47 $( \pm 7.28)$ and age is considered as one of the important predicting factor for balance impairment in diabetic patient. One study reported that age can be independent as well as cofounding factor in balance impairment. ${ }^{22}$ In our study 10 patients belong to the age group of 35-45 years, among them 8 patients were predisposed to medium risk of fall, 2 patients were at low risk of falling as compared to those 25 patients who were of age 56-65 years and among them 11 patients were at high risk, 10 were at medium risk and 4 patients were at low risk of falling. This interprets that fall risk increases with the advance age. These findings are supported by another study which documented that increase with one standard deviation in patient's age we might expect a decrease with 0.1 standard deviations in BBS score. ${ }^{23}$ The majority of the participants in our were at medium risk of fall who had diabetes for 10-20 years these findings were supported by another research which reported that patients who had diabetes for $>10$ years are duration longer than 10 years was associated with higher risk of fall and associated fractures. ${ }^{24}$ Female patients were at higher risk of fall as compared to men in our study. Anoter research reported that female gender increases the risk by $4.62 \% .25$

Results of our study concluded that majority of the patients (55.95) were at medium risk of fall. Our research findings were similar to another study which reported $84 \%$ were at medium risk of fall and $15 \%$ were at mild risk of fall among total 196 diabetic patients. ${ }^{26}$

The striking features of the present study are the utilization of validated instrument for the measurement of functional balance. Previous studies have largely focused on the dynamic balance, chiefly ignoring the importance of functional balance. Previous studies most commonly utilized Timed up and Go Test, Tineti Test, Functional Reach Test. 14, 27, ${ }^{28}$ Another study utilized Dynamic balance test and balance walk which were not validated and self-created tests. ${ }^{29}, 30$ Most of these balance tests utilized in the studies focused on anticipatory and reactive balance. Thus this study provides a framework for the practitioners to focus on the functional balance training of the patient, thus avoiding risk of fall. The major weak point of the study was that it was a case series and longer duration glycemic control was not evaluated. Future studies should be multicenter with heterogeneous representative population 


\section{Conclusion}

Greater numbers of patients with diabetic neuropathy have moderate risk of fall.

\section{References}

1. Zimmet P, Alberti KG, Magliano DJ, Bennett PH. Diabetes mellitus statistics on prevalence and mortality: facts and fallacies. Nature Reviews Endocrinology. 2016;12(10):616.

2. Saeedi P, Petersohn I, Salpea P, Malanda B, Karuranga $\mathrm{S}$, Unwin N, et al. Global and regional diabetes prevalence estimates for 2019 and projections for 2030 and 2045: Results from the International Diabetes Federation Diabetes Atlas. Diabetes research and clinical practice. 2019;157:107843.

3. Akhtar S, Nasir JA, Abbas T, Sarwar A. Diabetes in Pakistan: a systematic review and meta-analysis. Pakistan journal of medical sciences. 2019;35(4):1173.

4. Timar B, Popescu S, Timar R, Baderca F, Duica B, Vlad $M$, et al. The usefulness of quantifying intraepidermal nerve fibers density in the diagnostic of diabetic peripheral neuropathy: a cross-sectional study. Diabetology \& metabolic syndrome. 2016;8(1):31.

5. Van Dam PS, Cotter MA, Bravenboer B, Cameron NE. Pathogenesis of diabetic neuropathy: focus on neurovascular mechanisms. Eur. J. Pharmacol. 2013;719(1-3):180-6.

6. Akbari M, Dadgoo M, Ebrahimi I, Moradi S. Effects of Skin Stretch Sensory Stimuli on Balance in Patients with Diabetic Neuropathy. J Nov Physiother. 2011;1(105):2.

7. Mancini M, Horak FB. The relevance of clinical balance assessment tools to differentiate balance deficits. European journal of physical and rehabilitation medicine. 2010;46(2):239.

8. Hewston P, Deshpande N. Falls and balance impairments in older adults with type 2 diabetes: thinking beyond diabetic peripheral neuropathy. Can. J. Diabetes. 2016;40(1):6-9.

9. Silva P, Botelho PFFB, de Oliveira Guirro EC, Vaz MMO, de Abreu DCC. Long-term benefits of somatosensory training to improve balance of elderly with diabetes mellitus. Journal of bodywork and movement therapies. 2015;19(3):453-7.

10. Simoneau M, Teasdale N. Balance control impairment in obese individuals is caused by larger balance motor commands variability. Gait \& posture. 2015;41(1):203-8.

11. Joo Kim Y, Rogers JC, Kwok G, Dunn W, Holm MB. Somatosensation Differences in Older Adults with and Without Diabetes, and by Age Group. Occupational therapy in health care. 2016;30(3):231-44.

12. Grewal GS, Sayeed R, Schwenk M, Bharara M, Menzies $R$, Talal TK, et al. Balance rehabilitation: promoting the role of virtual reality in patients with diabetic peripheral neuropathy. J Am Podiatr Med Assoc. 2013;103(6):498507.
13. Morrison S, Colberg SR, Mariano M, Parson HK, Vinik Al. Balance training reduces falls risk in older individuals with type 2 diabetes. Diabetes care. 2010;33(4):748-50.

14. Vaz MM, Costa GC, Reis JG, Junior WM, de Paula FJA, Abreu DC. Postural control and functional strength in patients with type 2 diabetes mellitus with and without peripheral neuropathy. Archives of physical medicine and rehabilitation. 2013;94(12):2465-70.

15. Moslemi Haghighi F, Ghafarinejad F, Hemmati L, Saadat Z, Oorangi Z, Torabi S. Evaluation of ankle joint proprioception and balance in patients with type 2 diabetes and healthy subjects. Journal of Rehabilitation Sciences \& Research. 2015;2(1):17-9.

16. Downs S, Marquez J, Chiarelli P. The Berg Balance Scale has high intra-and inter-rater reliability but absolute reliability varies across the scale: a systematic review. Journal of physiotherapy. 2013;59(2):93-9.

17. Allet L, Armand S, De Bie R, Golay A, Pataky Z, Aminian $\mathrm{K}$, et al. Clinical factors associated with gait alterations in diabetic patients. Diabetic Medicine. 2009;26(10):1003-9.

18. Herman W, Pop-Busui R, Braffett B, Martin C, Cleary P, Albers J, et al. Use of the Michigan Neuropathy Screening Instrument as a measure of distal symmetrical peripheral neuropathy in type 1 diabetes: results from the Diabetes Control and Complications Trial/Epidemiology of Diabetes Interventions and Complications. Diabetic Medicine. 2012;29(7):937-44.

19. Beauchet O, Fantino B, Allali G, Muir S, Montero-Odasso $\mathrm{M}$, Annweiler C. Timed Up and Go test and risk of falls in older adults: a systematic review. J. Nutr. Health Aging. 2011;15(10):933-8.

20. Greenberg SA. Analysis of measurement tools of fear of falling for high-risk, community-dwelling older adults. Clinical Nursing Research. 2012;21(1):113-30.

21. Lee $\mathrm{I}-\mathrm{H}$, Park S-Y. Impairment of balance in elderly subjects with type 2 diabetes. Journal of physical therapy science. 2014;26(10):1519-20.

22. Brown SJ, Handsaker JC, Bowling FL, Boulton AJ, Reeves ND. Diabetic peripheral neuropathy compromises balance during daily activities. Diabetes Care. 2015;38(6):1116-22.

23. Timar B, Timar R, Gaiță L, Oancea C, Levai C, Lungeanu $D$. The impact of diabetic neuropathy on balance and on the risk of falls in patients with type 2 diabetes mellitus: a cross-sectional study. PloS one. 2016;11(4):e0154654.

24. Majumdar SR, Leslie WD, Lix LM, Morin SN, Johansson H, Oden A, et al. Longer duration of diabetes strongly impacts fracture risk assessment: the Manitoba BMD cohort. The Journal of Clinical Endocrinology \& Metabolism. 2016;101(11):4489-96.

25. Wettasinghe $A H$, Dissanayake DW, Allet $L$, Katulanda $P$, Lord SR. Falls in older people with diabetes: Identification of simple screening measures and explanatory risk factors. Primary care diabetes. 2020;14(6):723-8.

26. Ghazal J, Malik AN, Amjad I. Task oriented training improves the balance outcome \& reducing fall risk in diabetic population. Pak. J. Med. Sci. 2016;32(4):983. 
27. Najafi B, Armstrong DG, Mohler J. Novel wearable technology for assessing spontaneous daily physical activity and risk of falling in older adults with diabetes. SAGE Publications Sage CA: Los Angeles, CA; 2013.

28. Jernigan SD, Pohl PS, Mahnken JD, Kluding PM. Diagnostic accuracy of fall risk assessment tools in people with diabetic peripheral neuropathy. Physical therapy. 2012;92(11):1461-70.
29. Resnick HE, Stansberry KB, Harris TB, Tirivedi M, Smith $\mathrm{K}$, Morgan $\mathrm{P}$, et al. Diabetes, peripheral neuropathy, and old age disability. Muscle \& Nerve: Official Journal of the American Association of Electrodiagnostic Medicine. 2002;25(1):43-50.

30. Allet L, Armand S, De Bie R, Golay A, Monnin D, Aminian $K$, et al. The gait and balance of patients with diabetes can be improved: a randomised controlled trial. Diabetologia. 2010;53(3):458-66.

\section{Copyright Policy}

All Articles are made available under a Creative Commons "Attribution-NonCommercial 4.0

International" license. (https://creativecommons.org/licenses/by-nc/4.0/). Copyrights on any open access article published by Journal Riphah college of Rehabilitation Science (JRCRS) are retained by the author(s). Authors retain the rights of free downloading/unlimited e-print of full text and sharing/disseminating the article without any restriction, by any means; provided the article is correctly cited. JRCRS does not allow commercial use of the articles published. All articles published represent the view of the authors and do not reflect the official policy of JRCRS. 